\title{
A CHARACTERIZATION OF NONCOMMUTATIVE QUATERNION RINGS
}

\author{
CARL W. KOHLS
}

ABSTRACT. Noncommutative quaternion subrings of the ring of $4 \times 4$ matrices over a formally real field are characterized in terms of the form of commutators and the multiplicity of characteristic roots.

1. Introduction. From the proof of Lemma 10 in [2], one can easily deduce the following result: A subring $T$ of the ring of all $2 \times 2$ matrices over a formally real field $F$ is similar to a ring of matrices over the real closure of $F$ having the form $\left[\begin{array}{cc}a & b \\ -b & a\end{array}\right]$ if and only if every matrix in $T$ with a characteristic root in the real closure of $F$ is a scalar matrix. W. H. Reynolds suggested to the author that an analogous result leading to isomorphisms between rings of $4 \times 4$ matrices over $F$ and rings of quaternions would be worth seeking. We present such a theorem here for noncommutative rings. As might be expected, the situation is more involved than in the $2 \times 2$ case, so the characterization we obtain is somewhat different. In particular, the similarity transformation disappears.

It is a pleasure to thank the referee for his excellent suggestions, which led to concise proofs of Lemmas 1 and 3.

2. Preliminaries. The ring of all $4 \times 4$ matrices over an arbitrary formally real field $F$ will be denoted by $F_{4}$. We write $[A B]$ for the commutator $A B-B A$ of matrices $A$ and $B$ in $F_{4}$. The division ring $Q$ of quaternions over $F$ will be viewed as the set of elements in $F_{4}$ having the form

$$
\left[\begin{array}{rrrr}
a & b & c & d \\
-b & a & -d & c \\
-c & d & a & -b \\
-d & -c & b & a
\end{array}\right],
$$

and the opposite ring $Q^{\mathrm{op}}$ as the set of elements in $F_{4}$ having the form

Received by the editors October $1,1973$.

AMS (MOS) subject classifications (1970). Primary 15A30, 16A42; Secondary 15A18, 15A57, 16A40. 


$$
\left[\begin{array}{rrrr}
a & b & c & d \\
-b & a & d & -c \\
-c & -d & a & b \\
-d & c & -b & a
\end{array}\right] .
$$

Note that $Q^{\text {op }}$ is orthogonally similar to $Q$, through conjugation with the permutation matrix that interchanges the second and fourth columns. Also $Q$ commutes elementwise with $Q^{\mathrm{op}}$, and $Q \cap Q^{\mathrm{op}}$ is the ring of scalar matrices. Let $S$ denote the seven-dimensional subspace of $F_{4}$ consisting of all matrices that are the sum of a scalar matrix and a skew matrix. Clearly $Q \cup Q^{\mathrm{op}} \subseteq S$. By considering dimensions, one sees immediately that $S$ is the subspace of $\mathrm{F}_{4}$ generated by $Q \cup Q^{\mathrm{op}}$.

We shall be concerned with the following conditions on a subset $T$ of $F_{4}:$

(1) Each commutator of matrices in $T$ is skew.

(2) No matrix in $T$ has a simple characteristic root.

(3) There are two skew matrices in $T \cap Q$ or in $T \cap Q^{\text {op }}$ with nonzero commutator.

From the definition of $S$, each commutator of elements in $S$ is equal to a commutator of skew matrices, which is also skew. Thus, $S$ satisfies condition (1). It is easy to construct examples to show that $S$ does not satisfy condition (2), and that some subsets of $F_{4}$ disjoint from $S$ do satisfy it. However, by direct calculation one finds that the characteristic roots of the two matrices in $Q \cup Q^{\text {op }}$ with first row $(a b c d)$ are $a \pm\left(b^{2}+c^{2}+d^{2}\right)^{1 / 2} \sqrt{ }-1$, $a \pm\left(b^{2}+c^{2}+d^{2}\right)^{1 / 2} \sqrt{ }-1$. Hence $Q \cup Q^{\text {op }}$ satisfies condition (2).

3. The main result. We first prove three lemmas, from which the characterization follows rather quickly.

Lemma 1. If $T$ is a subset of $F_{4}$ that satisfies conditions (1) and (3), then $T \subseteq S$.

Proof. Choose skew matrices $A_{1}$ and $A_{2}$ in $T \cap Q$ or in $T \cap Q^{\mathrm{op}}$ with $\left[A_{1} A_{2}\right] \neq 0$. Let $B \in T$, and write $B=M+K$, where $M$ is symmetric and $K$ is skew. For $j=1$ and $2,\left[M A_{j}\right]$ is symmetric, $\left[K A_{j}\right]$ is skew, and $\left[M A_{j}\right]+$ $\left[K A_{j}\right]=\left[B A_{j}\right]$ is skew; hence $\left[M A_{j}\right]=0$. Let $r_{1}, r_{2}, r_{3}$ and $r_{4}$ be the characteristic roots of $M$ in the real closure of $F$, and select a nonzero vector $X_{i}$ in the characteristic subspace $V_{i}$ corresponding to $r_{i}(i=1,2,3,4)$. Since $\left[M A_{1}\right]=\left[M A_{2}\right]=0,\left\{X_{i}, A_{1} X_{i}, A_{2} X_{i}\right\} \subseteq V_{i}$ If $c_{0} X_{i}+c_{1} A_{1} X_{i}+$ $c_{2} A_{2} X_{i}=0$, where $c_{0}, c_{1}$ and $c_{2}$ are in the real closure of $F$, then $c_{1} A_{1}+$ 
$c_{2} A_{2}$ is skew with characteristic root $-c_{0}$, and is either invertible or zero, so $c_{0}=0$ and $c_{1} A_{1}+c_{2} A_{2}=0$; because $\left[A_{1} A_{2}\right] \neq 0$, this implies that $c_{1}=$ $c_{2}=0$. Hence the dimension of $V_{i}$ is at least three. Thus $V_{i} \cap V_{i+1} \notin\{0\}$ $(i=1,2,3)$, whence $r_{1}=r_{2}=r_{3}=r_{4}$. It follows that $M$ is a scalar matrix, and $B \in S$.

Lemma 2. If $B \in S$ and $\{B\}$ satisfies condition (2), then $B \in Q \cup Q^{\mathrm{op}}$.

Proof. Let $B=\left[b_{i j}\right]$. The characteristic polynomial of $B$, which is

$$
\left(b_{11}-x\right)^{4}+\left(\sum_{i<j} b_{i j}^{2}\right)\left(b_{11}-x\right)^{2}+\left(b_{12} b_{34}-b_{13} b_{24}+b_{14} b_{23}\right)^{2},
$$

must be the square of a quadratic polynomial in $b_{11}-x$. It follows quickly that

$$
\sum_{i<j} b_{i j}^{2}= \pm 2\left(b_{12} b_{34}-b_{13} b_{24}+b_{14} b_{23}\right) .
$$

The choice of a minus sign leads to

$$
\left(b_{12}+b_{34}\right)^{2}+\left(b_{13}-b_{24}\right)^{2}+\left(b_{14}+b_{23}\right)^{2}=0,
$$

so that

$$
b_{12}=-b_{34}, \quad b_{13}=b_{24}, \quad b_{14}=-b_{23},
$$

and $B \in Q$. Similarly, the choice of a plus sign yields $B \in Q^{\text {op }}$. Thus, $B \in Q \cup Q^{\text {op }}$.

Lemma 3. If $T$ is a subset of $Q \cup Q^{\mathrm{op}}$ that is closed under multiplication, then $T \subseteq Q$ or $T \subseteq Q^{\text {op }}$.

Proof. Suppose $T \not \subseteq Q^{\text {op }}$, and choose $B \in T$ with $B \notin Q^{\text {op }}$. Then $[A B] \neq$ 0 for some $A \in Q$. If $C \in T \cap Q^{\text {op }}$, either $C=0$ or $[A(B C)]=[A B] C \supsetneqq 0$. Since $B C \in Q \cup Q^{\text {op }}$, we have $B C \in Q$, whence $C \in Q$. Thus $T \subseteq Q$.

Theorem. A noncommutative subring $T$ of $F_{4}$ is a subring of either $Q$ or $Q^{\mathrm{op}}$ if and only if it satisfies conditions (1) and (2).

Proof. We observed in $\S 2$ that $S$ satisfies condition (1), and that $Q \cup$ $Q^{\text {op }}$ satisfies condition (2).

Conversely, assume that $T$ satisfies conditions (1) and (2). Choose $A$ and $B$ in $T$ with $C=[A B] \neq 0$. Then $C$ is skew, so by Lemma $2, C \in Q \cup$ $Q^{\text {op }}$. This implies that $C^{2}$ is a scalar matrix. If $[C A]=0$, then $C^{2}=$ $C[A B]=[A(C B)]$ is skew, so that $C^{2}=0$, whence $C=0$, a contradiction. Thus $D=[C A] \neq 0$ and $D$ is skew. By a similar argument $D \in Q \cup Q^{\mathrm{op}}$ and 
$[D C] \neq 0$. It follows that $\{C, D\} \subseteq T \cap Q$ or $\{C, D\} \subseteq T \cap Q^{\text {op }}$. Therefore $T$ satisfies condition (3). Lemmas 1,2 and 3 together now show that $T$ is a subring of either $Q$ or $Q^{\text {op }}$.

Remark 1. The observation that $C^{2}$ is a commutator is contained in the proof of The orem 18 in [1, Chapter 1]. Actually, the nilpotency of $C$ is an immediate consequence of that theorem, but we prefer to give a self-contained proof.

Remark 2. Obviously conditions (1) and (2) are also necessary for commutative subrings. But it is easy to see that they are not sufficient. For example, there are rings of diagonal matrices that satisfy them but are not even contained in $S$.

\section{REFERENCES}

1. Irving Kaplansky, Lie algebras and locally compact groups, Univ. of Chicago Press, Chicago, Ill., 1971. MR 43 \#2145.

2. C. W. Kohls and W. H. Reynolds, Embedding rings with a maximal cone and rings with an involution in quaternion algebras, Trans. Amer. Math. Soc. 176 (1973), 411-419. MR 47 \#1857.

DEPARTMENT OF MATHEMATICS, SYRACUSE UNIVERSITY, SYRACUSE, NEW YORK 13210 